\section{(6) OPEN ACCESS}

\title{
Alterations in the epithelial stem cell compartment could contribute to permanent changes in the mucosa of patients with ulcerative colitis
}

\author{
Isabella Dotti, ${ }^{1}$ Rut Mora-Buch, ${ }^{1}$ Elena Ferrer-Picón, ${ }^{1}$ Núria Planell, ${ }^{1,2}$ Peter Jung, ${ }^{3,4}$ \\ M Carme Masamunt, ${ }^{1}$ Raquel Franco Leal, ${ }^{1,5}$ Javier Martín de Carpi, ${ }^{6}$ Josep Llach, ${ }^{7}$ \\ Ingrid Ordás, ${ }^{1}$ Eduard Batlle, ${ }^{3,8}$ Julián Panés, ${ }^{1}$ Azucena Salas ${ }^{1}$
}

Additional material is published online only. To view please visit the journal online (http://dx.doi.org/10.1136/ gutjnl-2016-312609).

For numbered affiliations see end of article.

Correspondence to

Dr Azucena Salas, Department of Gastroenterology, IDIBAPS, Hospital Clínic, CIBERehd, 08036 Barcelona, Spain: asalas1@clinic.ub.es

Received 11 July 2016 Revised 12 September 2016 Accepted 18 September 2016 Published Online First 1 November 2016

\section{Linked}

- http://dx.doi.org/10.1136/ gutjnl-2016-313667

CrossMark

To cite: Dotti I, Mora-Buch $\mathrm{R}$ Ferrer-Picón $\mathrm{E}$, et al. Gut 2017:66:2069-2079.

\section{ABSTRACT}

Objective UC is a chronic inflammatory disease of the colonic mucosa. Growing evidence supports a role for epithelial cell defects in driving pathology. Moreover, long-lasting changes in the epithelial barrier have been reported in quiescent UC. Our aim was to investigate whether epithelial cell defects could originate from changes in the epithelial compartment imprinted by the disease.

Design Epithelial organoid cultures (EpOCs) were expanded ex vivo from the intestinal crypts of non-IBD controls and patients with UC. EpOCs were induced to differentiate (d-EpOCs), and the total RNA was extracted for microarray and quantitative real-time PCR ( $q P C R$ ) analyses. Whole intestinal samples were used to determine mRNA expression by qPCR, or protein localisation by immunostaining.

Results EpOCs from patients with UC maintained selfrenewal potential and the capability to give rise to differentiated epithelial cell lineages comparable with control EpOCs. Nonetheless, a group of genes was differentially regulated in the EpOCs and d-EpOCs of patients with UC, including genes associated with antimicrobial defence (ie, $L Y Z, P L A 2 G 2 A$ ), with secretory (ie, ZG16, CLCA1) and absorptive (ie, AQP8, MUC12) functions, and with a gastric phenotype (ie, ANXA10, CLDN18 and $L Y Z$ ). A high rate of concordance was found in the expression profiles of the organoid cultures and whole colonic tissues from patients with UC.

Conclusions Permanent changes in the colonic epithelium of patients with UC could be promoted by alterations imprinted in the stem cell compartment. These changes may contribute to perpetuation of the disease.

\section{INTRODUCTION}

$\mathrm{UC}$ is a chronic remitting and relapsing IBD, which is restricted to the colonic and rectal mucosa. ${ }^{1}$ The mucosal surface is covered by a single layer of epithelial cells, which separate the intestinal lumen from the underlying lamina propria and protect the host from harmful luminal components ${ }^{2}$ while allowing for nutrient transport. ${ }^{3}$ The epithelial layer is composed of millions of invaginations called crypts. At the base of the crypt, a small number of multipotent adult stem cells constantly give rise to all of the differentiated epithelial

\section{Significance of this study}

What is already known on this subject?

- UC is a chronic inflammatory condition characterised by periods of activity followed by phases of endoscopic remission and mucosal healing.

- During UC remission, multiple biological pathways remain deregulated despite endoscopic and histological recovery. Several are associated with epithelial cell functions.

- The ex vivo organoid culture system is a promising tool for understanding the pathophysiology of the intestinal epithelium.

\section{What are the new findings?}

- Expression differences are maintained in ex vivo expanded epithelial organoid cultures generated from biopsy samples of patients with UC compared with non-IBD subjects. These differences are possibly due to permanent changes in the stem cell compartment.

- Among the genes permanently deregulated in organoid cultures from patients with UC, several are associated with epithelial secretory and absorptive functions.

- The expression signature detected in organoid cultures derived from patients with UC shows traits characteristic of the upper $\mathrm{Gl}$ tract.

How might it impact on clinical practice in the foreseeable future?

- The organoid culture system can serve as a model to explore the presence of lasting alterations in the intestinal epithelium of patients with IBD and to investigate their roles in the perpetuation of the disease.

subsets. ${ }^{4}$ Under homeostatic conditions, it is estimated that the entire epithelium is completely renewed every 4-5 days. ${ }^{5}$

During the active phase of UC, the release of proinflammatory factors contributes to the damage and destruction of the epithelial layer, ${ }^{6}{ }^{7}$ which causes exposure of the underlying lamina propria to luminal antigens. In order to re-establish 
homeostasis, the epithelial layer undergoes an intense process of regeneration through mechanisms that are just beginning to be understood. $^{8} 9$ Nonetheless, a previous study by our group revealed that the intestinal epithelium of patients with UC in remission shows an expression profile that is significantly different from that found in healthy mucosa. ${ }^{10}$ These data strongly support the hypothesis that changes in the epithelium persist long after inflammation has been resolved and epithelial integrity has been re-established.

Given the rapid turnover of the epithelial compartment and the presence of sustained changes in the colonic epithelium of patients with quiescent UC, we asked whether these alterations were driven by a sustained deregulation of the epithelial cell compartment rather than by signals coming from the microenvironment.

In order to address this question, we generated epithelial organoid cultures (EpOCs) from non-IBD controls and patients with UC. ${ }^{11}$ By whole genome expression analysis, we identified key functions (eg, secretory and antimicrobial mechanisms) that persist deregulated in the epithelium of patients with UC. Our results suggest that lasting changes in the epithelial compartment may permanently influence its homeostatic functions in patients with UC, potentially contributing to disease progression.

\section{METHODS}

\section{Patient population}

Patients with an established diagnosis of UC of at least 3 months in duration, and non-IBD controls (Ctrl) were included in the study after obtaining written informed consent. Non-IBD controls were those subjects undergoing surgery for colorectal cancer (CRC), colonoscopy for mild GI symptoms or screening for CRC (none of these controls presented colonic lesions). Online supplementary table S1A shows the clinical and demographic characteristics of the study subjects. Samples were distributed among different subgroups on the basis of the experimental approaches used. Patients were recruited at the Department of Gastroenterology, Hospital Clinic Barcelona. A cohort of adult non-IBD controls and patients with Crohn's disease (CD) (recruited at Hospital Clinic Barcelona, see online supplementary table $\mathrm{S} 1 \mathrm{~B}$ ) was used to generate sigmoid EpOCs and evaluate, in CD-derived EpOCs, the expression changes detected in UC-derived EpOCs. Additional EpOCs from small intestinal biopsy samples (SInt-EpOCs) from the above cohort and from paediatric subjects (recruited at Hospital Sant Joan de Deu, Barcelona, and not included in online supplementary table S1) were generated to determine the expression of regionspecific markers. The study protocol was approved by the Ethics Committees of Hospital Clinic Barcelona and Hospital Sant Joan de Deu.

\section{Assessment of disease activity}

Endoscopic UC activity at the time of the colonoscopy was categorised according to the Mayo endoscopic subscore. ${ }^{12}$ Active UC was defined as a Mayo endoscopic subscore of $\geq 1$; quiescent UC was defined as a Mayo score of 0 in a previously involved segment. Endoscopic CD remission was defined as a SES-CD $\leq 2$ for the segment from which the biopsies were obtained.

\section{Sample collection}

Biopsy samples (see online supplementary table S1A) were collected during routine colonoscopy from non-IBD controls and patients with UC or CD with no evidence of IBD-associated dysplasia or neoplasia, as assessed by chromoendoscopy. Biopsy samples from patients with UC or CD were collected from previously involved or mildly inflamed sigmoid mucosa based on endoscopic criteria. Surgical samples from patients with UC were obtained after colectomy performed for refractoriness to medical treatment. Additional tissue sections from the healthy small intestine and stomach were provided by the Biobank of IDIBAPS upon approval by the ethics committee.

\section{Epithelial organoid culture}

Samples from non-IBD controls or patients with UC (Patient Group 1, see online supplementary table S1A, B) were used for generating EpOCs. Crypts embedded in Matrigel (BD Biosciences, San Jose, California, USA) were cultured and passaged as previously described. ${ }^{11}$ EpOCs were then induced to differentiate into differentiated EpOCs (d-EpOCs). The absence of contamination by cells of non-epithelial origin in organoid cultures was assessed by immunofluorescence using antivimentin (Leica Biosystems, Wetzlar, Germany), anti-CD3 (Vector Laboratories, Burlingame, Alabama, USA), and anti-CD45 (BD Pharmingen, San Jose, California, USA) antibodies (see online supplementary figure $\mathrm{S} 1$ ).

\section{RNA isolation}

Total RNA from first-passage EpOCs (Patient Group 1, see online supplementary table S1A,B) and biopsy samples (Patient Group 2, see online supplementary table S1A) previously placed in RNAlater RNA Stabilization Reagent (Qiagen, Hilden, Germany) was isolated using the RNeasy Kit (Qiagen).

\section{Microarray analysis}

For microarray analysis, paired first-passage EpOC and d-EpOC generated from colonic samples of 11 non-IBD controls and 8 patients with UC were used (Patient Group 1, see online supplementary table S1A). The derived cRNA was hybridised to high-density oligonucleotide Affymetrix GeneChip Human Genome U219 Array (Affymetrix, Santa Clara, California, USA). Raw data were analysed using Bioconductor tools in R (V.3.1.0). Microarray raw data (CEL files) and processed data have been deposited in NCBI's Gene Expression Omnibus and are accessible through GEO Series accession no GSE75916 (URL: http://www. ncbi.nlm.nih.gov/geo/query/acc.cgi?token = ehejqkoobraxxip \& acc $=$ GSE75916).

\section{Quantitative real-time PCR}

Total RNA (samples from Patient Group 1 or 2, see online supplementary table S1A, and Patient Group 1, online supplementary table $\mathrm{S} 1 \mathrm{~B}$ ) was transcribed to cDNA using reverse transcriptase (High Capacity cDNA Archive RT kit, Applied Biosystems, Carlsbad, California, USA). Quantitative real-time PCR (qPCR) was performed in an ABI PRISM 7500 Fast RT-PCR System (Applied Biosystems) using predesigned TaqMan Assays (Applied Biosystems).

\section{Immunostaining}

Immunostaining of organoid and tissue sections (Groups 1 and 3, see online supplementary table S1A) was performed using commercially available antibodies. Image acquisition and overlay was performed on an Olympus BX51 microscope (Tokyo, Japan) using CellF Software.

\section{Statistical analysis}

Quantitative data are expressed as the mean and range, and categorical variables as absolute frequencies. A Wilcoxon test was performed to examine statistically different expression patterns 
between two groups, and a Kruskal-Wallis test was performed to examine statistical significance in multiple group data sets, followed by a pairwise Wilcoxon test with Bonferroni-Holm correction for multiple testing. A p value of $<0.05$ was considered statistically significant. Data were analysed using R (V.3.1.0) (published online first: 2014, http://www.r-project.org/).

More detailed information is described in online supplementary materials and methods.

\section{RESULTS}

EpOCs from patients with UC can be efficiently expanded and differentiated ex vivo

In order to generate ex vivo stem cell-enriched EpOCs, crypt units from the distal colon of non-IBD controls and patients with UC (Patient Group 1, see online supplementary table S1A) were used. EpOCs from control and UC samples showed similar self-renewal capacity (figure 1A) and comparable histological and molecular features (figure 1B). A thin monolayer of non- polarised cells showing mRNA expression of the stem cell marker LGR5 and the proliferation antigen KI-67, and low expression of epithelial differentiation markers (ie, L-FABP and mucin-2, markers of absorptive and mucus-secreting cells, respectively) was observed in both cases. Withdrawal of specific factors from the stem medium drove differentiation of EpOCs to d-EpOCs within 5 days (figure 1A). d-EpOCs from both non-IBD controls and patients with UC presented marked nuclear polarisation towards the basolateral side, as well as the appearance of vacuolar structures oriented towards the luminal side. A marked decrease in $L G R 5^{+}$and $\mathrm{KI}-67^{+}$cells and an increased number of $\mathrm{L}_{-} \mathrm{FABP}^{+}$and mucin- $2^{+}$cells were observed (figure 1B).

To further explore the mechanisms of in vitro differentiation of EpOCs from non-IBD controls and patients with UC, a whole genome microarray analysis was performed. Principal component analysis separated EpOC from d-EpOC populations, regardless of sample origin (figure 2A). Approximately 4500 probes

A

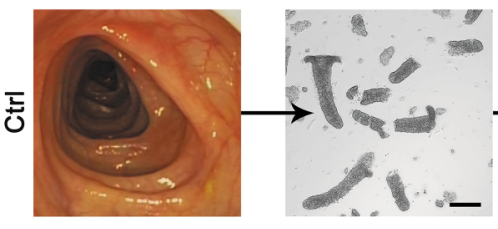

1. Sample collection

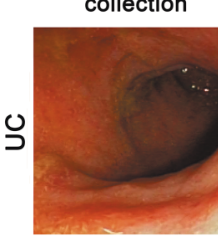

2. Crypt isolation

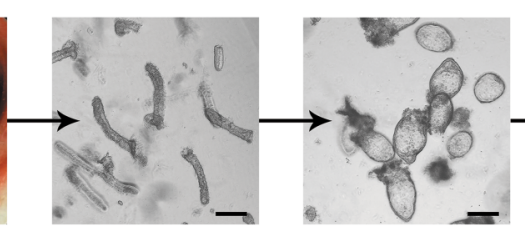

3. Crypt culture

4. EpOC
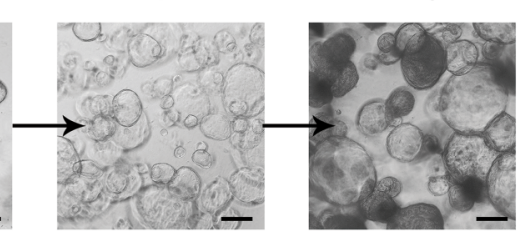

B

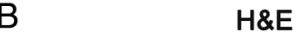

H\&E

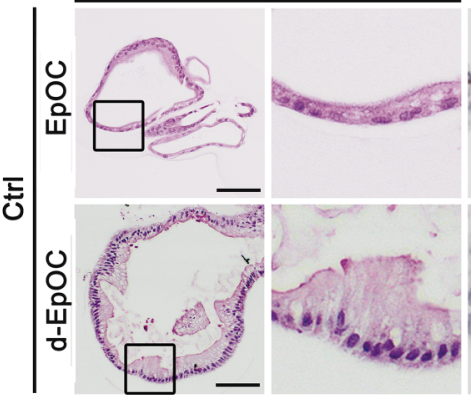

LGR5

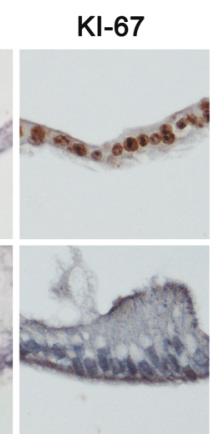

L-FABP
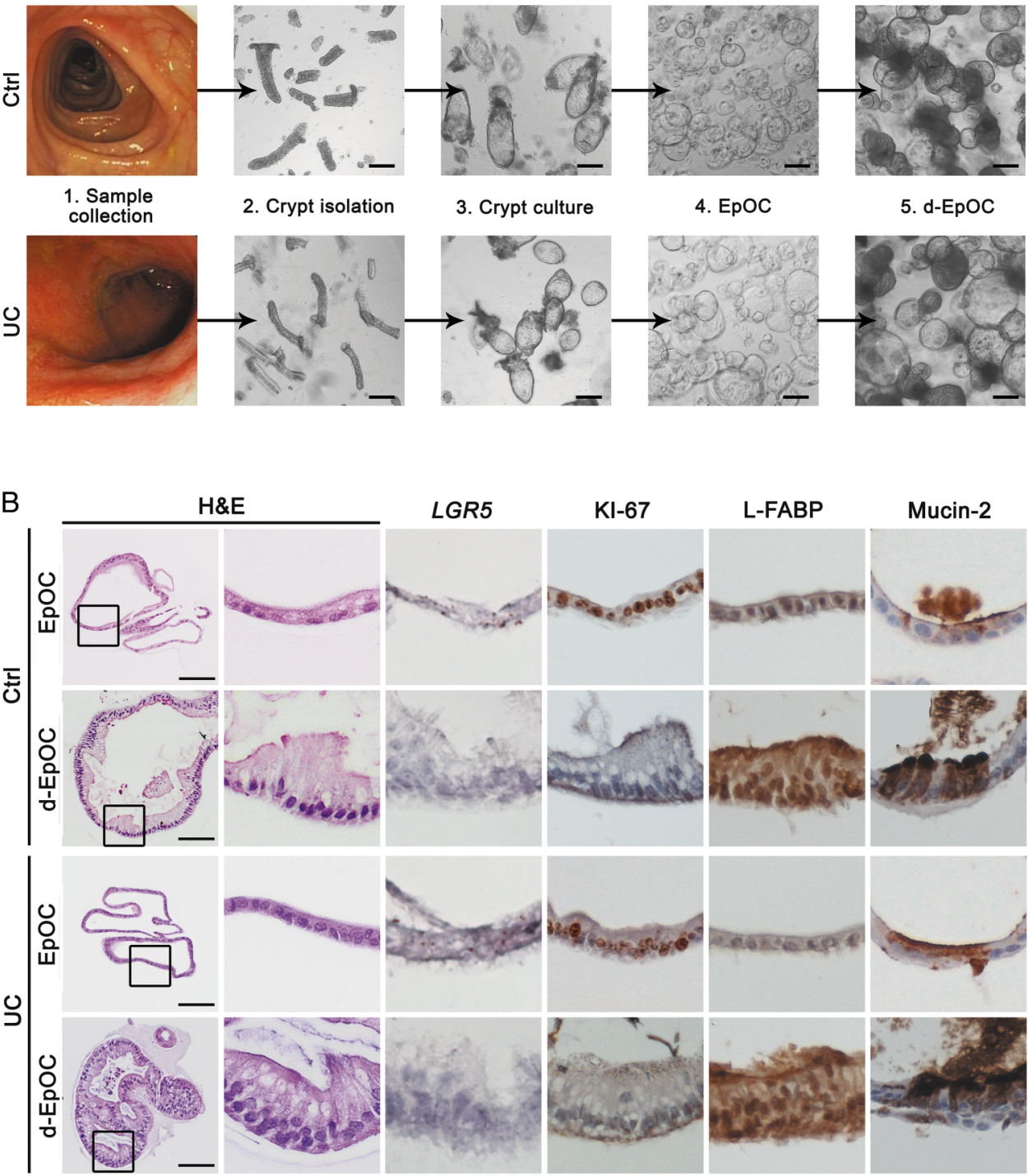

5. d-EpOC
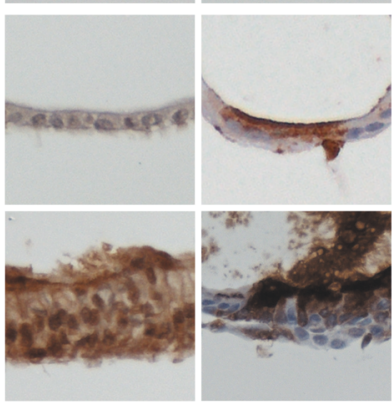

Figure 1 Colonic crypts from non-IBD controls and patients with UC give rise to epithelial organoid cultures (EpOCs) in vitro. (A) Schematic representation of colonic crypt culture and expansion of EpOCs from non-IBD controls and patients with UC. Upon differentiation, EpOCs terminally differentiated into d-EpOCs within 5 days. Scale bar: $200 \mu \mathrm{m}$. (B) Morphological characterisation of EpOCs and differentiated EpOCs (d-EpOCs) from non-IBD controls and patients with UC. Representative H\&E staining, immunochemical staining of antigen KI-67, L-FABP and mucin-2 proteins, and in situ hybridisation to detect $L G R 5$ mRNA are shown. Scale bars: $100 \mu \mathrm{m}$. 
A

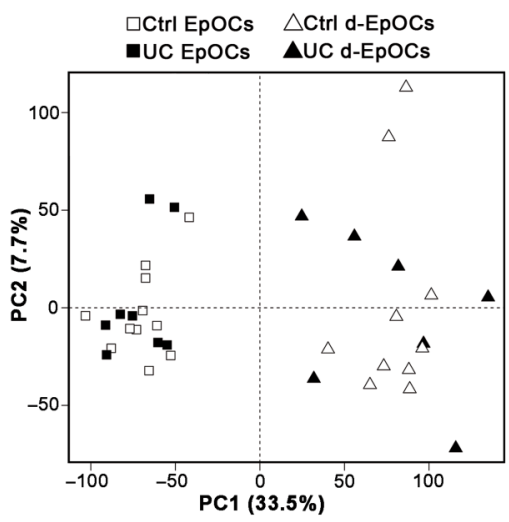

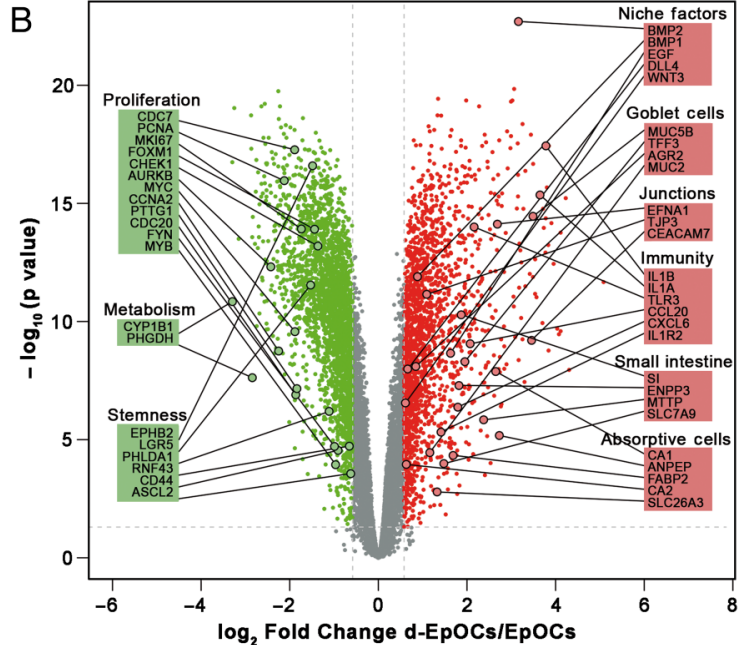

Figure 2 Epithelial organoid cultures (EpOCs) generated from non-IBD controls and patients with UC follow comparable differentiation programmes. (A) Principal component analysis (PCA) of microarray-based genome-wide gene expression data derived from EpOCs and differentiated EpOCs (d-EpOCs) generated from non-IBD controls and patients with UC. A two-principal component plot is shown with the first component along the $\mathrm{X}$-axis and the second along the $\mathrm{Y}$-axis. The proportion of explained variance is shown for each component. (B) Volcano plot showing the changes in expression between d-EpOCs and EpOCs regardless of patient origin (ie, non-IBD controls or patients with UC). The log-base2 fold-change from d-EpOCs versus EpOCs is shown on the X-axis and the -log-base10 of adjusted $\mathrm{p}$ value on the $\mathrm{Y}$-axis. Dots in green and red represent genes that are significantly downregulated or upregulated $(|\mathrm{FC}| \geq 1.5$ (log2 fold-change $\geq 0.58)$ and adjusted $\mathrm{p}$ value $\leq 0.05)$ in $\mathrm{d}$-EpOCs compared with EpOCs, respectively. The genes with no significant expression differences are represented in grey. Several differentially expressed genes have been highlighted and grouped according to their involvement in relevant epithelial cell functions.

were found to be differentially expressed (false discovery rate (FDR) $\leq 0.05$ and $|\mathrm{FC}| \geq 1.5$ ) (figure $2 \mathrm{~B}$ ). In particular, EpOCs showed significantly higher expression of the well-described stem cell markers EPHB2, LGR5 and ASCL2, and the proliferation markers MKI67, MYC and PTTG1. Interestingly, EpOCs showed increased expression of CYP1B1, as well as PHGDH, two previously undescribed markers of colonic stem cells. In d-EpOCs, a significant upregulation of intestinal markers of absorptive enterocytes (eg, CA1, FABP2 and CA2) and mucous-secreting goblet cells (eg, MUC5B, TFF3 and AGR2) was observed. d-EpOCs also showed increased expression of several regulators of the immune response, including the IL-1R type II, in agreement with our recent findings. ${ }^{9}$ In addition, d-EpOCs displayed an upregulation of WNT3, EGF, DLL4 and BMP2 niche factors. Technical validation of a set of stemness, proliferation and epithelial differentiation markers was performed by qPCR (see online supplementary figure S2A). Differentiation of EpOCs also induced significant upregulation of genes characteristic of the small intestinal epithelium (ie, SI, ENPP3, MTTP and SLC7A9) (figure 2B). Nonetheless, SInt-EpOCs, compared with colonderived EpOCs, maintained higher expression of the small intestine-specific APOA4, DEFA5 and LYZ genes, and lower expression of the colon-specific CLCA4 and MUC5B genes (see online supplementary figure $\mathrm{S} 2 \mathrm{~B}$ ).

In summary, we show that ex vivo expanded EpOCs, regardless of whether the samples originated from non-IBD controls or patients with UC, are indistinguishable in their ability to generate differentiated cell lineages.

\section{A specific expression signature characterises EpOCs and d-EpOCs from patients with UC compared with non-IBD controls}

Despite the identification of a common programme of EpOC differentiation, specific transcriptional signatures for EpOCs and d-EpOCs from patients with UC compared with non-IBD controls were determined by differential expression analysis (figure 3A). Eighty-four genes were differentially regulated within EpOCs (UC/Ctrl EpOCs) and 77 within d-EpOCs (UC/ Ctrl d-EpOCs), with 24 of those genes common to both organoid populations $(\mathrm{p} \leq 0.05$ and $|\mathrm{FC}| \geq 1.5$ ) (figure $3 \mathrm{~B}$ and see online supplementary table S2).

qPCR analysis confirmed the significant upregulation of transcripts for the antimicrobial protein lysozyme $\mathrm{C}(L Y Z)$, the tight junction protein claudin-18 (CLDN18), annexin-10 (ANXA10), hyal-1 (HYAL1) and serpin B2 (SERPINB2) in EpOCs and d-EpOCs obtained from patients with UC compared with non-IBD controls. In contrast, mRNA encoding for the water transporter aquaporin-8 (AQP8), the mucus-secreting cell markers hZG16 (ZG16) and hCLCA1 (CLCA1), the transmembrane mucin-12 (MUC12) and the antimicrobial GIIC sPLA2 (PLA2G2A) were confirmed to be significantly downregulated in d-EpOCs from patients with UC compared with controls (figure 3C). In addition, increased lysozyme $\mathrm{C}$ protein concentration (see online supplementary figure $\mathrm{S3A}$ ), as well as enzymatic activity (see online supplementary figure S3B) in organoid culture supernatants from patients with UC compared with non-IBD controls, was observed. The concentration of hZG16 in culture supernatants of EpOC and d-EpOC cultures generated from patients with UC was significantly lower compared with non-IBD controls (see online supplementary figure S3A).

Next, we determined whether expression of the panel of genes deregulated in UC-derived EpOCs was also differentially expressed in CD-derived organoid cultures. An independent cohort of sigmoid EpOCs and d-EpOCs from non-IBD controls $(n=6)$ and $C D$ patients $(n=8)$ was generated and transcriptional changes were assessed by qPCR. Out of the 10 genes analysed, we only detected a statistically significant change in the mRNA expression of lysozyme $\mathrm{C}$ in EpOCs and d-EpOCs of patients with CD compared with non-IBD controls (figure 4).

Thus far, we provide novel evidence that EpOCs and d-EpOCs obtained from patients with UC exhibit a differential expression profile compared with non-IBD controls, suggesting 

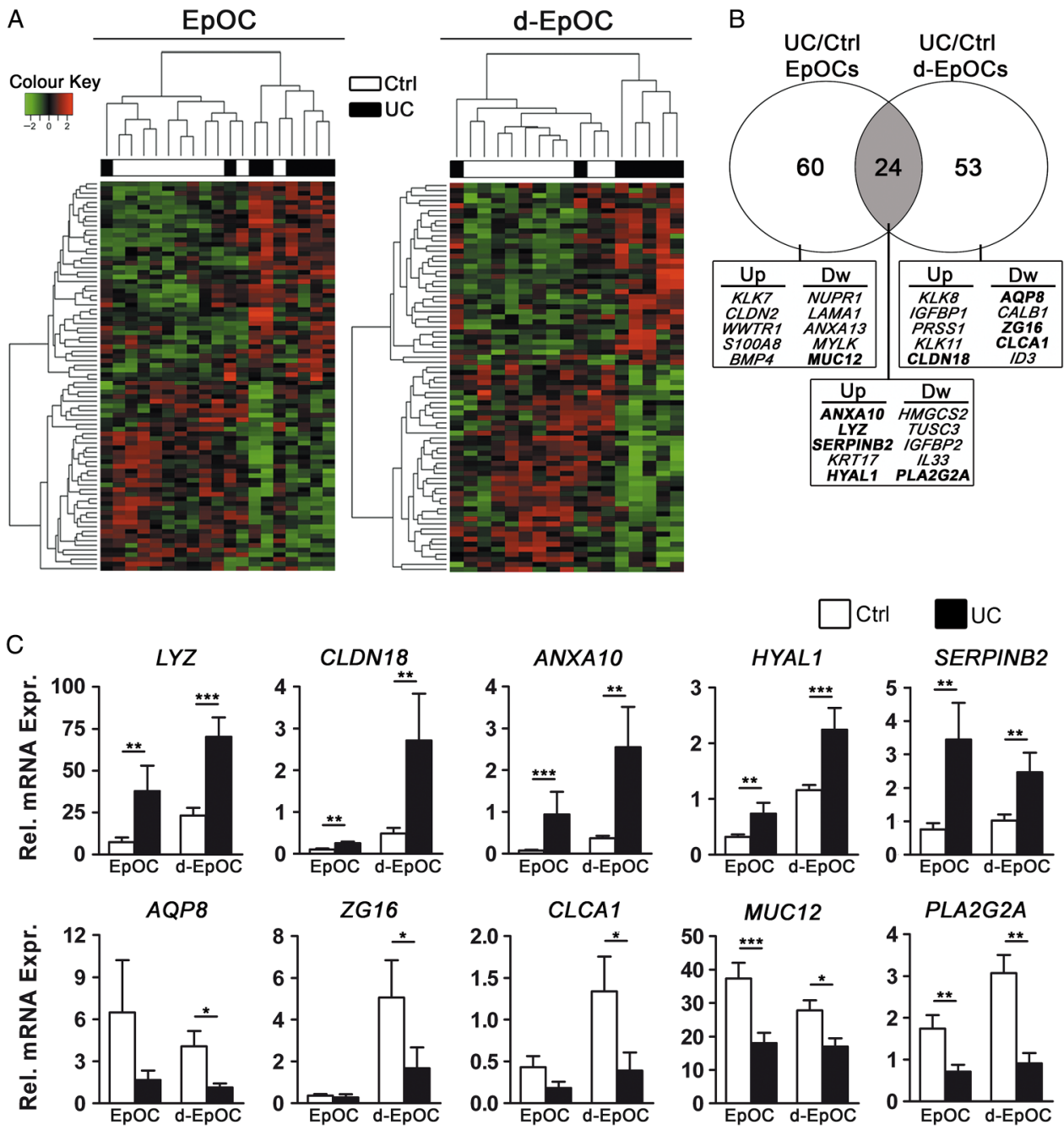

Figure 3 Differential gene expression profiles in epithelial organoid cultures (EpOCs) and differentiated EpOCs (d-EpOCs) generated from non-IBD controls compared with patients with UC. (A) Heat map representations of differentially expressed genes ( $p$ value $\leq 0.05$ and $|F C| \geq 1.5$ ) in patients with UC compared with non-IBD controls. Differentially expressed genes in EpOCs and d-EpOCs are shown separately. Each row depicts one individual probe and each column an experimental sample. High expression levels are shown in red and low expression levels in green. An unsupervised hierarchical cluster method, using a Pearson distance and average linkage method, was applied for both gene and sample classification. Samples from non-IBD controls (white, $n=11$ EpOCs and $n=10 \mathrm{~d}$-EpOCs) and patients with UC (black, $n=8$ EpOCs and $n=7 d-E p 0 C s$ ) are shown. (B) Venn diagram showing the number of genes differentially expressed between UC and non-IBD EpOCs under stem-enriched and differentiated conditions. The overlap, in grey, shows differentially expressed genes shared between the two data sets. A selection of upregulated and downregulated genes is shown (genes validated by quantitative real-time PCR (qPCR) are depicted in bold) (C) Relative mRNA levels by qPCR of selected genes found differentially expressed in the microarray analysis (samples included in the microarray analysis have been used). Messenger RNA levels are relative to ACTB expression. Mean \pm SEMs. ${ }^{*} p<0.05,{ }^{* *} p<0.01,{ }^{* *} p<0.001$ by one-tailed Wilcoxon test.

that there are sustained differences within the epithelial stem cell compartment of the UC mucosa.

\section{Intact tissue epithelium and organoid cultures from patients} with UC show common expression features

Next, we asked how the deregulated genes found in the organoid cultures of patients with UC were modulated during inactive and active phases of the disease. To answer this, we aligned the EpOC and d-EpOC expression profile with our previously published transcriptional signature from biopsies of non-IBD controls and patients with UC (series accession number GSE38713). ${ }^{10}$ Approximately, 66\% (72/109) of genes significantly upregulated or downregulated in EpOCs and/or d-EpOCs from patients with UC were found to be similarly deregulated in whole biopsies from patients with UC (see online supplementary table S2). Interestingly, expression of the
EpOC-associated transcriptional signature in non-involved segments from patients with UC was comparable with that of non-IBD controls (data not shown). To further validate these results, we performed qPCR expression analysis of a selected panel of genes in colonic biopsies from non-IBD controls and patients with UC in endoscopic remission or with active disease (Patient Group 2, see online supplementary table S1A). LYZ, CLDN18 and HYAL1 transcripts analysed in the mucosa of patients with active UC followed a pattern of upregulation similar to that observed in UC-derived organoid cultures (figures $3 \mathrm{C}$ and $5 \mathrm{~A}$ ). Changes in the protein expression were analysed by immunohistochemical staining in colonic sections (Patient Group 3, see online supplementary table S1A). Lysozyme C was barely detected in epithelial cells in control non-IBD sigmoid mucosa (figure 5B). However, it markedly accumulated in the granules of secretory epithelial cells along 

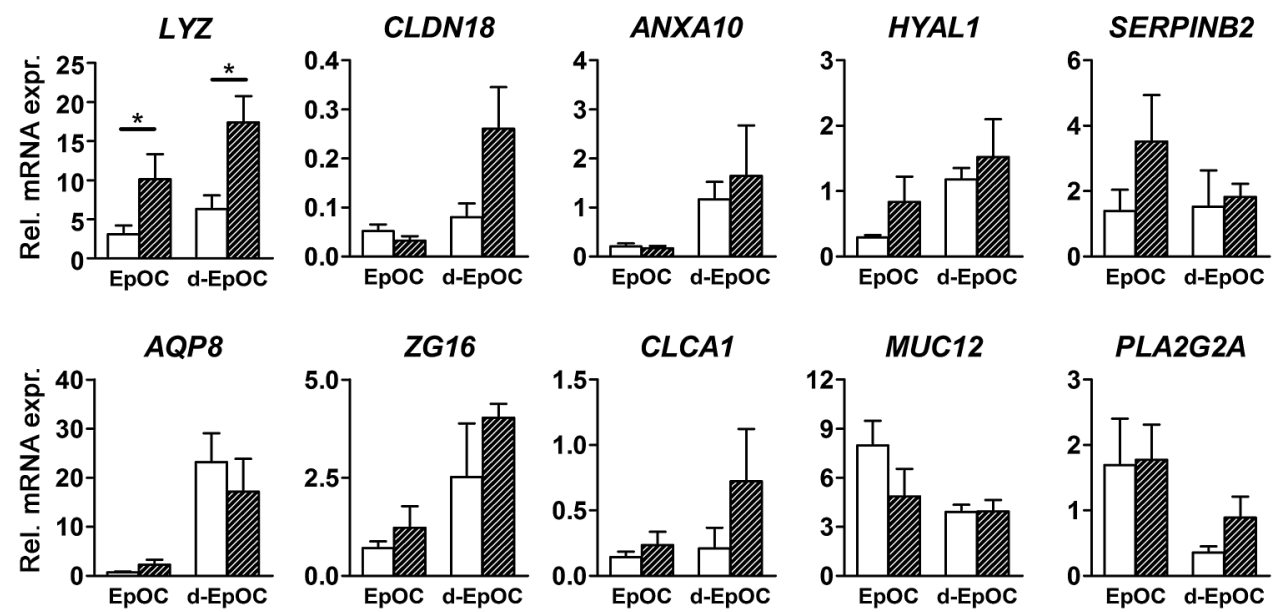

Figure 4 mRNA expression analysis in Crohn's disease (CD)-derived epithelial organoid cultures (EpOCs) of genes differentially expressed in UC-derived EpOCs. The mRNA expression analysis of the selected panel of genes described in figure $3 \mathrm{C}$ was performed in an independent cohort of EpOCs and differentiated EpOCs (d-EpOCs) generated from sigmoid biopsies of non-IBD controls $(n=6)$ and patients with $C D(n=8)$. The organoid cultures were generated and analysed using the procedures described in the Methods section. Gene expression is relative to ACTB gene.

Mean \pm SEMs. ${ }^{*} p<0.05,{ }^{* *} p<0.01,{ }^{* * *} p<0.001$ by one-tailed Wilcoxon test.

the crypts in samples of active UC (and in metaplastic Paneth cell clusters not observed in figure 5B). Additional cell sources of lysozyme, probably macrophages and activated dendritic cells, ${ }^{13}{ }^{14}$ were detected in the stroma of both control and inflamed mucosa. Similarly, claudin-18, which was not detectable in healthy colonic mucosa, showed sporadic expression in active UC samples. Remarkably, the expression of lysozyme C and claudin-18 was also found to be deregulated in samples from patients with UC in remission. No substantial alterations in hyal-1 protein expression were observed in samples from patients with UC compared with controls. EpOCs and d-EpOCs and tissue samples from patients with UC also shared a panel of downregulated genes (see online supplementary table S2). In particular, transcriptional downregulation of ZG16 in colonic samples mirrored the expression results observed in organoid cultures from patients with UC (figures 3C and 6A). Similarly, a downregulation of hZG16 protein expression was detected in sporadic crypts of samples from patients with active and inactive UC (figure 6B) (Patient Group 3, see online supplementary table S1A). CLCA1 did not exhibit significant transcriptional changes in tissue samples from patients with UC compared with controls (figure 6A). Nonetheless, a loss of hCLCA1 protein expression was sporadically observed in the epithelium of samples from patients with UC (figure 6B). PLA2G2A showed increased transcription in tissue samples from patients in remission and active UC compared with controls (figure 6A). A decrease in GIIC sPLA2 protein expression was occasionally observed in the upper UC crypts compared with the control sections, despite the marked aberrant expression in metaplastic Paneth cells (figure 6B). Costaining of lysozyme C, claudin-18, hyal-1, hZG16, hCLCA1 and GIIC sPLA2 with the epithelial marker Ep-CAM in colonic sections from patients with UC confirmed the epithelial localisation of the selected proteins in tissue (see online supplementary figure $\mathrm{S} 4$ ).

Here, we provide evidence that the differences observed in the EpOCs from patients with UC mimic those detected in tissue epithelial cells, further supporting the possible contribution of lasting epithelial alterations in the perpetuation of the disease.
The expression signature of UC-derived EpOCs and d-EpOCs suggests the acquisition of an upper $\mathrm{Gl}$ tract profile

Thus far, our data suggest the presence of sustained changes in UC-derived EpOCs. We therefore wondered whether the global gene expression signature of organoid cultures derived from patients with UC resembled the profile of other regions of the GI tract. To this end, we aligned the expression signature of EpOCs and d-EpOCs from patients with UC to the previously published gene expression map of the healthy human GI tract (series accession number GSE10867). ${ }^{15}$ Interestingly, genes perturbed in EpOCs and/or d-EpOCs from patients with UC compared with controls ( $n=100$ genes) were able to segregate the GI samples according to their location (figure 7), revealing a considerable number of genes associated with different GI regions (see online supplementary table S2). Genes overexpressed in EpOCs and/or d-EpOCs from patients with UC (left column, in purple) correlated with a gene subset highly expressed mainly in healthy antrum, jejunum and duodenum. Among them, the identified genes LYZ, CLDN18, HYAL1 and ANXA10 could be observed. Similarly, genes downregulated in EpOCs and/or d-EpOCs from patients with UC (left column, in orange) were transcripts predominantly expressed in healthy colon (ie, AQP8, ZG16 and CLCA1). Immunohistochemical staining confirmed that both lysozyme $\mathrm{C}$ and claudin-18 were abundantly expressed by healthy gastric epithelium (see online supplementary figure S5). As expected, lysozyme $\mathrm{C}$ also showed selective expression by Paneth cells in healthy small intestinal mucosa (similarly to GIIC sPLA2). In contrast, protein expression of hZG16, hCLCA1 and GIIC sPLA2 was undetectable in healthy gastric epithelium. No significant deregulation of hyal-1 expression was evident in healthy gastric epithelium compared with control or UC-affected sigmoid colon.

Overall, our data suggest that UC-derived epithelium acquires traits of the upper GI tract while losing functional capacities characteristic of the distal intestinal epithelium.

\section{DISCUSSION}

In the present study, we provide evidence that lasting alterations in the epithelium of patients with UC could be due, at least 
A

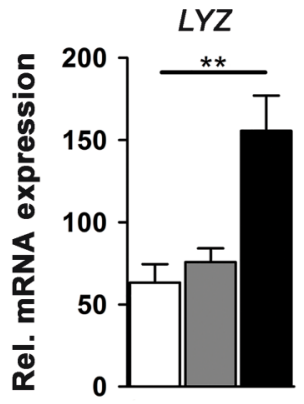

Ctrl

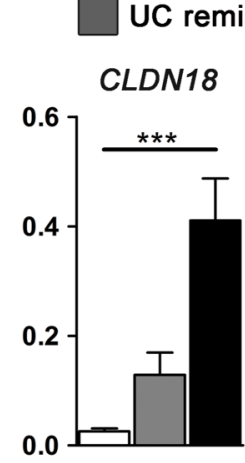

UC active

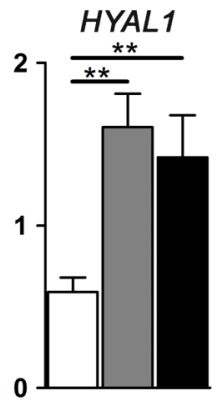

B

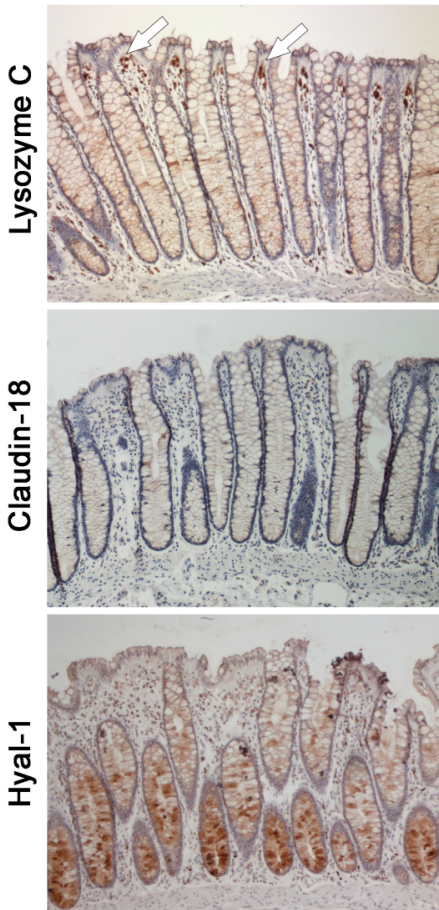

Ctrl
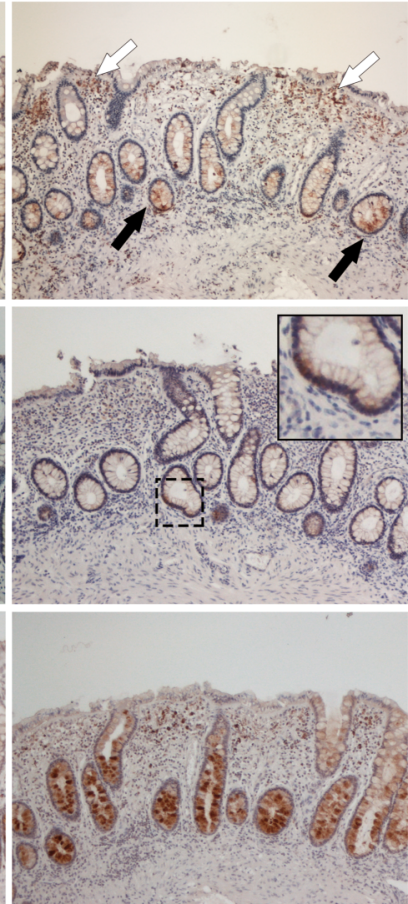

UC remission
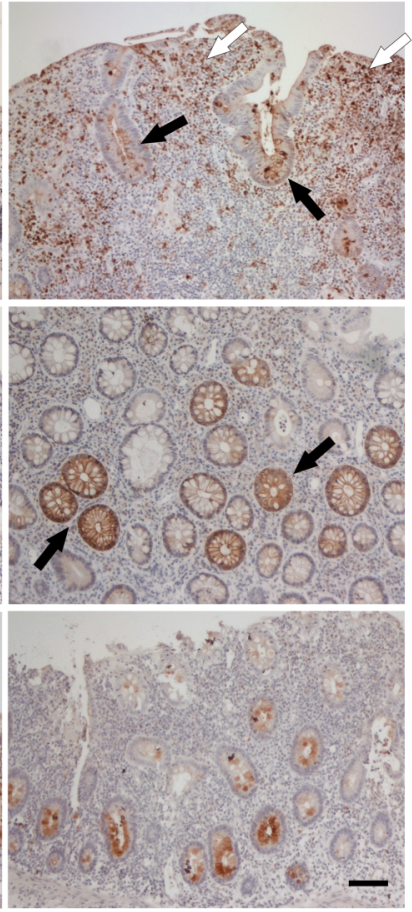

UC active

Figure 5 Epithelial cells from involved colonic UC tissue share a panel of upregulated genes with UC epithelial organoid cultures (EpOCs) and differentiated EpOCs. (A) Relative mRNA expression by quantitative real-time PCR of selected genes previously found to be overexpressed in UC compared with control EpOCs. Sigmoid mucosa biopsies from non-IBD controls $(n=13)$ were compared with biopsies from patients with UC in remission ( $n=24)$ and with active UC $(n=11)$. Messenger RNA levels are relative to ACTB expression. Mean \pm SEMs. ${ }^{*} p<0.05,{ }^{* *} p<0.01,{ }^{* * *} p<0.001$ by pairwise Wilcoxon test with Bonferroni-Holm correction for multiple testing. (B) Tissue immunohistochemical staining of selected genes differentially expressed in UC compared with control EpOCs. Sigmoid mucosa samples from non-IBD controls were compared with samples from patients with inactive and active UC. Black arrows indicate protein upregulation in UC epithelium compared with the control samples. White arrowheads show the expression of lysozyme $C$ in additional cells in the lamina propria. Scale bar: $100 \mu \mathrm{m}$.

partially, to changes in the stem cell compartment. Our results shed new light on a long-standing question in the field of chronic intestinal inflammation: whether epithelial cells can acquire a programme that impacts disease progression or whether they are mere bystanders to the ongoing pathological process.

Several genetic polymorphisms associated with UC risk localise within active regulatory elements in intestinal epithelial cells. ${ }^{16}$ These results support the hypothesis that defective epithelial cell function may be driving UC pathogenesis. Moreover, we recently described that epithelial cells from the involved colonic mucosa of patients with UC acquire a unique transcriptional signature that is maintained long after acute inflammation is resolved, suggesting a permanent epithelial cell dysfunction. ${ }^{10}$ While this study reveals a potential mechanism for
UC-associated complications such as CRC, it has not yielded any information on whether these changes are driven by signals coming from the surrounding environment or whether they are imprinted in the epithelial compartment.

In the present work, we addressed this issue by taking advantage of a recently established approach for expanding and maintaining intestinal epithelial cells ex vivo as 3D organoid cultures. ${ }^{11}{ }^{17}$ Using this system, we first showed that colonic crypts from non-IBD controls and patients with UC were able to expand as stem cell-enriched EpOCs with highly comparable efficiency. Moreover, no apparent alterations in the homeostatic balance between the stem, proliferative and differentiated epithelial cell populations were observed. d-EpOCs, regardless of the sample origin, acquired characteristics of differentiated intestinal absorptive and secretory cell lineages, as already 
A

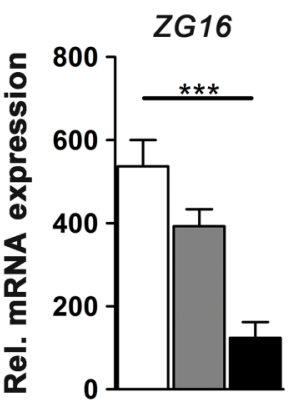

Ctrl

UC remission

CLCA1

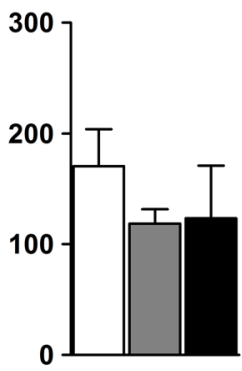

UC active

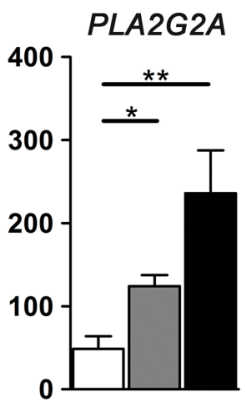

B

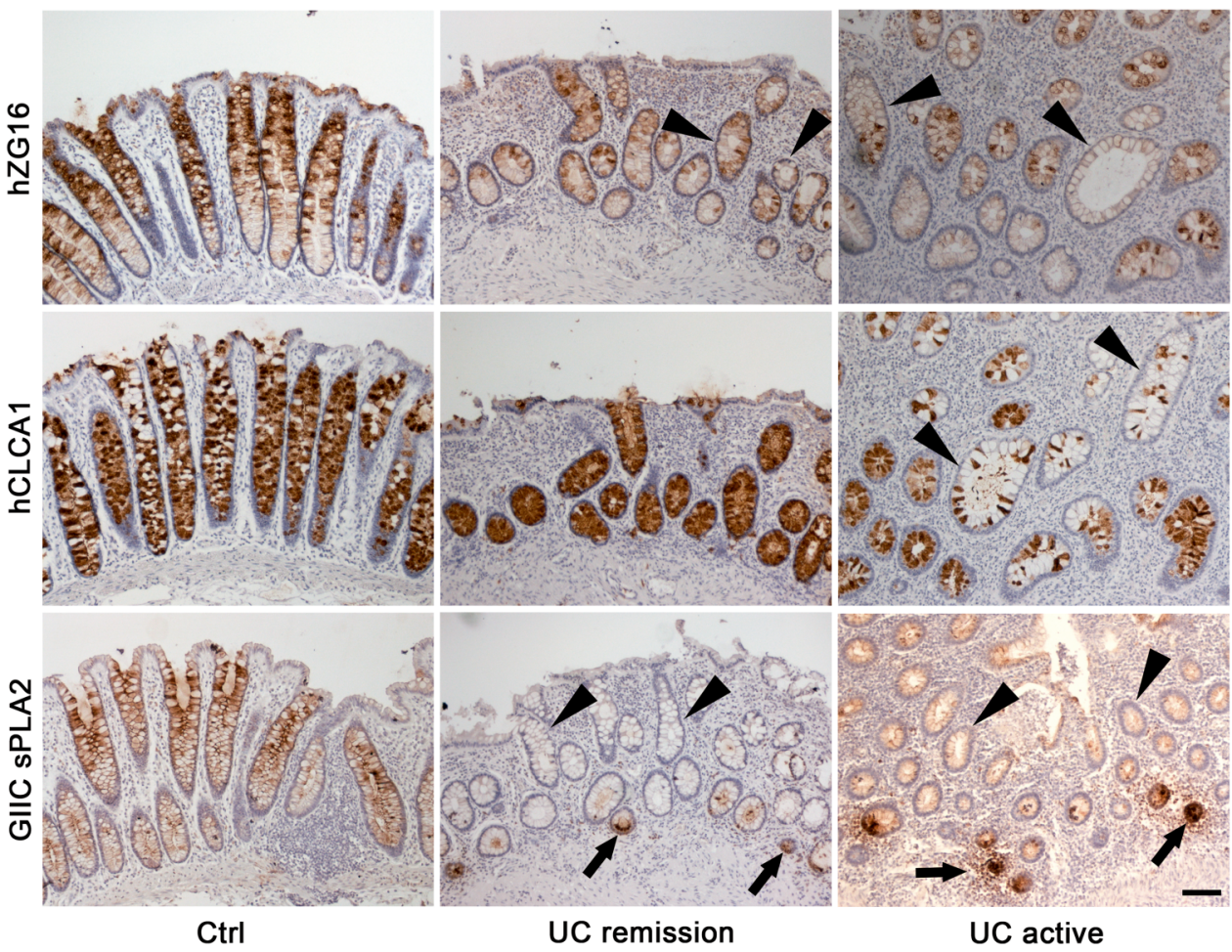

Figure 6 Epithelial cells from involved colonic UC tissue share a panel of downregulated genes with UC epithelial organoid cultures (EpOCs) and differentiated EpOCs. (A) Relative mRNA expression by quantitative real-time PCR of selected genes previously found to be downregulated in UC compared with control EpOCs. Sigmoid mucosa biopsies from non-IBD controls $(n=13)$ were compared with biopsies from patients with UC in remission ( $n=24)$ and with active UC $(n=11)$. Messenger RNA levels are relative to ACTB expression. Mean \pm SEMs. ${ }^{*} p<0.05,{ }^{* *} p<0.01,{ }^{* * *} p<0.001$ by pairwise Wilcoxon test with Bonferroni-Holm correction for multiple testing. (B) Tissue immunohistochemical staining of selected genes differentially expressed in UC compared with control EpOCs. Sigmoid mucosa samples from non-IBD controls were compared with samples from patients with inactive and active UC. Black arrows and black arrowheads indicate protein upregulation and downregulation in UC epithelium compared with non-IBD controls, respectively. Scale bar: $100 \mu \mathrm{m}$.

described. ${ }^{18} 19$ Upon differentiation, upregulation of WNT3, EGF, DLL4 and BMP2, important niche factors primarily assigned to murine Paneth cells in the small intestine, ${ }^{20}$ was observed. We also provide further evidence that supports the ability of expanded EpOCs to maintain the location-specific expression of small intestine versus colon markers when cultured and differentiated in vitro. ${ }^{21} 22$

Despite the overall similarities, we identified a set of genes that exhibited sustained differential expression in organoid cultures derived from patients with UC. The fact that these changes were observed in EpOCs derived from UC samples in remission or mildly active implies that at least some aspects of the previously described imprinting in the UC epithelium ${ }^{10}$ could be explained by permanent alterations in the stem cell compartment.

Interestingly, deregulation of gene expression in EpOCs correlated with changes in the involved mucosal samples of patients with UC, thus strengthening the significance of our results in ex vivo cultures. Conversely, the expression of this set of genes showed no differences between non-involved areas from patients with UC and healthy regions from non-IBD controls. This observation further supports the hypothesis that defects in intestinal epithelium may be acquired during the course of the disease and can contribute to its perpetuation over time. Moreover, analysis of the transcriptional signature suggested the acquisition of upper GI traits by UC-derived epithelial cells. 


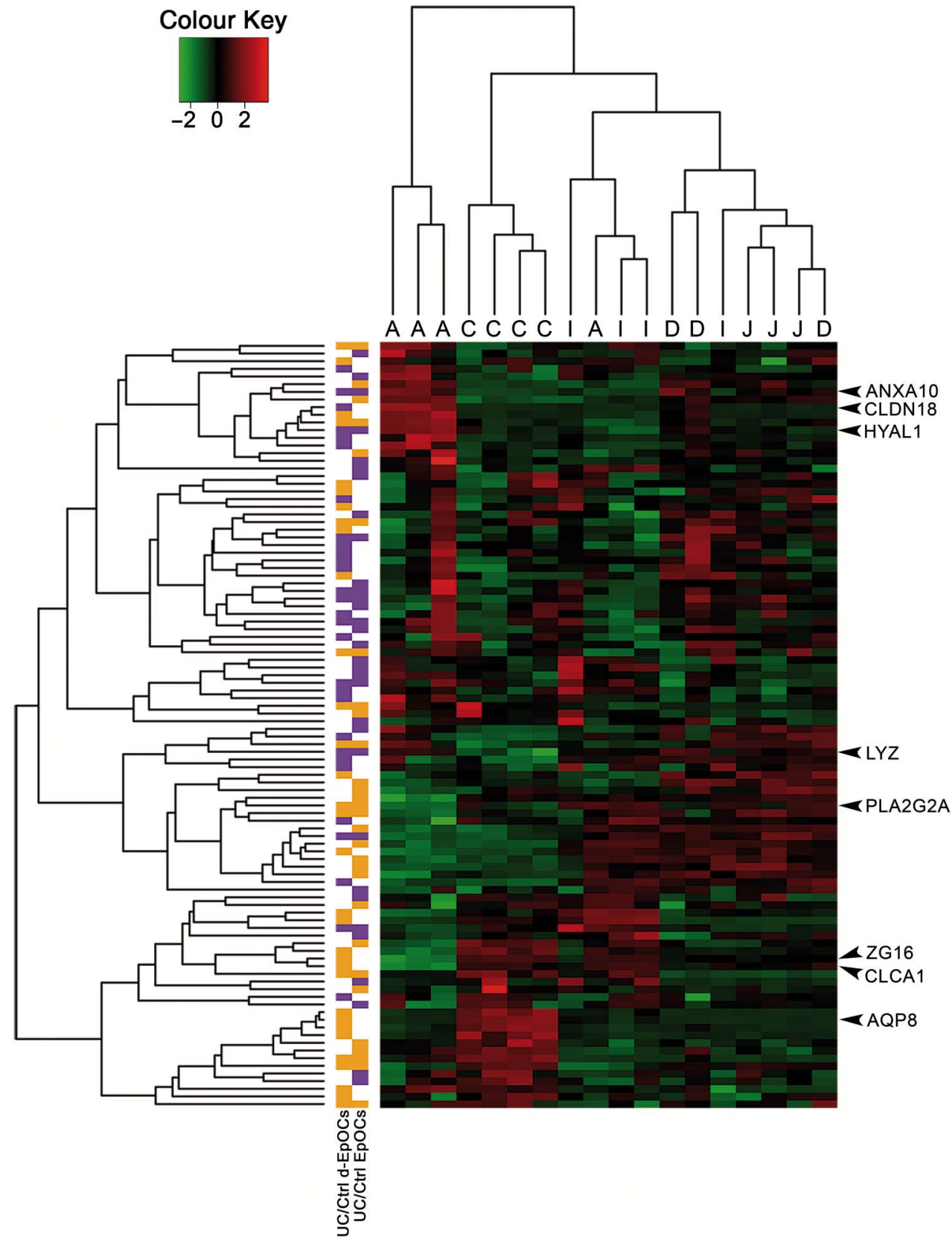

Figure 7 Epithelial organoid cultures (EpOCs) and differentiated EpOCs (d-EpOCs) from patients with UC adopt expression characteristics of the upper GI tract. Heat map representation of the expression of genes identified in the UC-derived EpOCs shown in figure 3A and B in a set of biopsy samples originating from different segments of the GI tract (C: colon; I: ileum; J: jejunum; D: duodenum; A: antrum). Each row shows one individual probe and each column an experimental sample. High expression levels are shown in red and low expression levels in green. An unsupervised hierarchical cluster method, using a Pearson distance and average linkage method, was applied for both gene and sample classification. In the left columns, genes upregulated or downregulated in UC versus non-IBD control organoid cultures are shown in purple or orange, respectively.

The signature in UC-derived epithelial cells included the aberrant expression of LYZ, CLDN18, ANXA10 and HYAL1. Lysozyme $C$, an enzyme that degrades the peptidoglycan of several Gram-positive bacteria, ${ }^{23}$ is primarily expressed in small intestinal Paneth cells and gastric epithelium. Ectopic lysozyme $\mathrm{C}$ expression in the colonic epithelium of patients with UC is commonly associated with metaplastic Paneth-like cells. ${ }^{24}$ In addition, here we identify secretory cells along the UC crypt, which provide an additional source of lysozyme C. The overexpression of LYZ in EpOCs derived from patients with UC and CD could explain, at least in part, the importance of this antimicrobial protein in the persistence of dysbiosis in both forms of IBD. ${ }^{25}$ Claudin-18 is a component of tight junctions that in physiological conditions is uniquely expressed by the gastric and pulmonary epithelium. ${ }^{26}$ Ectopic expression of claudin-18 has been associated with experimental colitis and human UC. ${ }^{27}$ Moreover, it has been correlated with the serrated pathway of CRC. ${ }^{28}$ Similarly, annexin-A10, which serves diverse physiological cell functions almost exclusively in gastric epithelium, ${ }^{29}$ has been recently identified as a marker of gastric phenotype in serrated pathway of CRC. ${ }^{30}$ These observations support the hypothesis that UC-derived epithelial cells can become predisposed to UC-associated CRCs. ${ }^{10}$ 
UC-derived epithelial cells showed a marked downregulation of genes associated with epithelial functions of the distal GI tract. This was the case with hZG16 and hCLCA1, two major components of the mucus produced mainly by secreting cells of the distal digestive tract. ${ }^{31}$ hZG16 is involved in the recognition of pathogens through its binding to glycosaminoglycans and mannose, ${ }^{32}$ while hCLCA1 is a $\mathrm{Ca}^{2+}$ channel with putative functions in the innate immunity modulation. ${ }^{33}$ Overall, these data provide evidence of a lasting epithelial defect in mucus barrier quality, a feature that has already been shown to correlate with UC. 3435

In addition, our results suggest an overall defect in the absorptive function of UC-derived epithelial cells. In particular AQP8, which encodes an epithelial water-transporting protein abundantly expressed by the absorptive cells in the colon, ${ }^{36}$ was found markedly downregulated in UC EpOCs, in line with previous observations in tissue samples. ${ }^{10}{ }^{37}$ We also found a significant downregulation of MUC12 in EpOCs derived from patients with UC. This transmembrane mucin, which is part of the enterocyte glycocalyx, ${ }^{31}$ has been previously associated with UC. ${ }^{38}$ Interestingly, previous findings showed a reduction in AQP8 and MUC12 expression in CRC. ${ }^{39} 40$ These observations corroborate an association between permanent changes in the epithelium of patients with UC and colon neoplasia. GIIC sPLA2 is a bactericidal protein expressed in small intestinal Paneth cells ${ }^{41}$ and, to a lesser extent, in colonic epithelial cells, ${ }^{42}$ as confirmed by our observations. We detected a downregulation of GIIC sPLA2 in secretory cells along the UC crypts compared with control sections, thus supporting the results in organoid cultures. The ectopic expression of GIIC sPLA2 in metaplastic Paneth cells in tissue samples from patients with UC, as observed by others, ${ }^{43}$ could be explained by the transitory effects of external stimuli (eg, lipopolysaccharide) on intestinal epithelium. ${ }^{44}$ The downregulation of PLA2G2A in secretory cells, with the concomitant overexpression of $L Y Z$, supports the idea of a permanent rearrangement of the antimicrobial functions in UC-derived epithelial cells.

In light of our results, key questions remain regarding the molecular mechanisms driving these expression changes in UC-derived epithelium and their functional consequences. While these questions are beyond the scope of the present study, it is tempting to speculate that epigenetic changes may contribute to expression alterations of the UC epithelium. In particular, DNA methylation changes in the UC epithelium have been already linked to UC pathogenesis and its complications. ${ }^{45-47}$ Finally, we propose that lasting changes in the UC epithelium may translate into alterations in the absorptive and secretory functions, which could help perpetuate the disease over time.

\author{
Author affiliations \\ 'Department of Gastroenterology, IDIBAPS, Hospital Clínic, CIBERehd, Barcelona, \\ Spain \\ ${ }^{2}$ Bioinformatics Platform, CIBERehd, Barcelona, Spain \\ ${ }^{3}$ Oncology Program, Institute for Research in Biomedicine (IRB), Barcelona, Spain \\ ${ }^{4}$ German Cancer Consortium (DKTK), Heidelberg, Germany \\ ${ }^{5}$ IBD Research Laboratory, Surgery Department, Universidade Estadual de Campinas, \\ Campinas, Sao Paulo, Brazil \\ ${ }^{6}$ Department of Gastroenterology, Hepatology and Pediatric Nutrition, Hospital Sant \\ Joan de Deu, Barcelona, Spain \\ ${ }^{7}$ Endoscopy Unit, Hospital Clínic, CIBERehd, Barcelona, Spain \\ ${ }^{8}$ Institució Catalana de Recerca i Estudis Avançats (ICREA), Barcelona, Spain
}

Acknowledgements We thank the Biobank Facility at the Institut d'Investigacions Biomèdiques August Pi i Sunyer (IDIBAPS) for their support, the Endoscopy and Pathology Departments at Hospital Clinic Barcelona and Hospital Sant Joan de Déu in Barcelona for providing us with the samples required to conduct this study, and our patients for their selfless participation. We are indebted to Joe Moore for editorial assistance.

Contributors ID designed and conducted experiments, acquired and analysed data, and wrote the manuscript. RM-B, EF-P and RFL designed and conducted experiments. NP executed bioinformatics and biostatistics analysis. MCM, JMdC, JL, 10 and JP recruited patients and/or collected samples. PJ and EB designed and supervised experiments. AS designed the study, supervised experiments, analysed data and wrote the manuscript.

Funding This work was supported by grants BFU2012-35999/BFI, MC1_TV3_122931, PIE13/00033 and the Helmsley Trust. RM-B. was a recipient of an FPI fellowship from the Ministerio de Economía y Competitividad (BES-2010-030033). EF-P was a recipient of an FI-DGR 2015 fellowship from AGAUR (Generalitat de Catalunya). RFL was supported by a research fellowship from CAPES-Brazil. This work is cofinanced by the European Union through the European Regional Development Fund (ERDF), 'A way of making Europe'. NP is supported by the Centro de Investigación Biomédica en Red de Enfermedades Hepáticas y Digestivas (CIBERehd).

Competing interests None declared.

\section{Patient consent Obtained.}

Ethics approval Hospital Clínic and Sant Joan de Deu Ethical Committees, Barcelona, Spain.

Provenance and peer review Not commissioned; externally peer reviewed.

Data sharing statement The transcriptional data of the EpOCs and d-EpOCs generated from non-IBD controls and patients with UC have been deposited in the Gene Expression Omnibus at the National Center for Biotechnology Information (NCBI) with accession no. GSE75916.

Writing assistance English language assistance by Joe Moore was funded by the Leona and Harry Helmsley Charitable Trust.

Open Access This is an Open Access article distributed in accordance with the Creative Commons Attribution Non Commercial (CC BY-NC 4.0) license, which permits others to distribute, remix, adapt, build upon this work non-commercially, and license their derivative works on different terms, provided the original work is properly cited and the use is non-commercial. See: http://creativecommons.org/ licenses/by-nc/4.0/

\section{REFERENCES}

1 Ordás I, Eckmann L, Talamini M, et al. Ulcerative colitis. Lancet 2012;380:1606-19.

2 Johansson ME, Gustafsson JK, Holmén-Larsson J, et al. Bacteria penetrate the normally impenetrable inner colon mucus layer in both murine colitis models and patients with ulcerative colitis. Gut 2014;63:281-91.

3 Mowat AM, Agace WW. Regional specialization within the intestinal immune system. Nat Rev Immunol 2014;14:667-85.

4 Vermeulen L, Snippert HJ. Stem cell dynamics in homeostasis and cancer of the intestine. Nat Rev Cancer 2014;14:468-80

5 Vereecke L, Beyaert R, van Loo G. Enterocyte death and intestinal barrier maintenance in homeostasis and disease. Trends Mol Med 2011;17:584-93.

6 Nava P, Koch S, Laukoetter MG, et al. Interferon-gamma regulates intestinal epithelial homeostasis through converging beta-catenin signaling pathways. Immunity 2010;32:392-402.

7 Su L, Nalle SC, Shen L, et al. TNFR2 activates MLCK-dependent tight junction dysregulation to cause apoptosis-mediated barrier loss and experimental colitis. Gastroenterology 2013;145:407-15.

8 Miyoshi H, Ajima R, Luo CT, et al. Wnt5a potentiates TGF- $\beta$ signaling to promote colonic crypt regeneration after tissue injury. Science 2012;338:108-13.

9 Mora-Buch R, Dotti I, Planell N, et al. Epithelial IL-1R2 acts as a homeostatic regulator during remission of ulcerative colitis. Mucosal Immunol 2016;9:950-9.

10 Planell N, Lozano JJ, Mora-Buch $\mathrm{R}$, et al. Transcriptional analysis of the intestinal mucosa of patients with ulcerative colitis in remission reveals lasting epithelial cell alterations. Gut 2013;62:967-76.

11 Jung $P$, Sato T, Merlos-Suárez $A$, et al. Isolation and in vitro expansion of human colonic stem cells. Nat Med 2011;17:1225-7.

12 Schroeder KW, Tremaine WJ, Ilstrup DM. Coated oral 5-aminosalicylic acid therapy for mildly to moderately active ulcerative colitis. A randomized study. N Engl J Med 1987;317:1625-9.

13 Fahlgren A, Hammarström S, Danielsson A, et al. Increased expression of antimicrobial peptides and lysozyme in colonic epithelial cells of patients with ulcerative colitis. Clin Exp Immunol 2003;131:90-101.

14 Bonnardel J, Da Silva C, Henri S, et al. Innate and adaptive immune functions of Peyer's patch monocyte-derived cells. Cell Rep 2015;11:770-84.

15 Comelli EM, Lariani S, Zwahlen MC, et al. Biomarkers of human gastrointestina tract regions. Mamm Genome 2009;20:516-27. 
16 Mokry M, Middendorp S, Wiegerinck $\mathrm{CL}$, et al. Many inflammatory bowel disease risk loci include regions that regulate gene expression in immune cells and the intestinal epithelium. Gastroenterology 2014;146:1040-7.

17 Sato $T$, Stange DE, Ferrante $M$, et al. Long-term expansion of epithelial organoids from human colon, adenoma, adenocarcinoma, and Barrett's epithelium. Gastroenterology 2011;141:1762-72.

18 Jung P, Sommer C, Barriga FM, et al. Isolation of Human Colon Stem Cells Using Surface Expression of PTK7. Stem Cell Reports 2015;5:979-87.

19 VanDussen KL, Marinshaw JM, Shaikh N, et al. Development of an enhanced human gastrointestinal epithelial culture system to facilitate patient-based assays. Gut 2015:64:911-20.

20 Sato T, van Es JH, Snippert HJ, et al. Paneth cells constitute the niche for Lgr5 stem cells in intestinal crypts. Nature 2011;469:415-18.

21 Middendorp S, Schneeberger $\mathrm{K}$, Wiegerinck $\mathrm{CL}$, et al. Adult stem cells in the small intestine are intrinsically programmed with their location-specific function. Stem Cells 2014;32:1083-91.

22 Cramer JM, Thompson T, Geskin A, et al. Distinct human stem cell populations in small and large intestine. PLOS ONE 2015;10:e0118792.

23 Obita T, Ueda T, Imoto T. Solution structure and activity of mouse lysozyme M. Cell Mol Life Sci 2003;60:176-84.

24 Mitsuhashi J, Mikami T, Saigenji K, et al. Significant correlation of morphological remodeling in ulcerative colitis with disease duration and between elevated p53 and p21 expression in rectal mucosa and neoplastic development. Pathol Int 2005:55:113-21.

25 Matsuoka K, Kanai T. The gut microbiota and inflammatory bowel disease. Semin Immunopathol 2015:37:47-55.

26 Sanada Y, Oue N, Mitani Y, et al. Down-regulation of the claudin-18 gene, identified through serial analysis of gene expression data analysis, in gastric cancer with an intestinal phenotype. J Pathol 2006;208:633-42.

27 Zwiers A, Fuss IJ, Leijen $\mathrm{S}$, et al. Increased expression of the tight junction molecule claudin-18 A1 in both experimental colitis and ulcerative colitis. Inflamm Bowel Dis 2008:14:1652-9.

28 Sentani K, Sakamoto N, Shimamoto F, et al. Expression of olfactomedin 4 and claudin-18 in serrated neoplasia of the colorectum: a characteristic pattern is associated with sessile serrated lesion. Histopathology 2013;62:1018-27.

29 Lu SH, Yuan RH, Chen YL, et al. Annexin A10 is an immunohistochemical marker for adenocarcinoma of the upper gastrointestinal tract and pancreatobiliary system. Histopathology 2013;63:640-8.

30 Tsai JH, Lin YL, Cheng YC, et al. Aberrant expression of annexin A10 is closely related to gastric phenotype in serrated pathway to colorectal carcinoma. Mod Pathol 2015:28:268-78.
31 Pelaseyed T, Bergström JH, Gustafsson JK, et al. The mucus and mucins of the goblet cells and enterocytes provide the first defense line of the gastrointestinal tract and interact with the immune system. Immunol Rev 2014;260:8-20.

32 Tateno $H$, Yabe $R$, Sato $T$, et al. Human ZG16p recognizes pathogenic fungi through non-self polyvalent mannose in the digestive system. Glycobiology 2012;22:210-20.

33 Ching JC, Lobanova L, Loewen ME. Secreted hCLCA1 is a signaling molecule that activates airway macrophages. PLOS ONE 2013:8:e83130.

34 Pullan RD, Thomas GA, Rhodes $M$, et al. Thickness of adherent mucus gel on colonic mucosa in humans and its relevance to colitis. Gut 1994;35:353-9.

35 Strugala V, Dettmar PW, Pearson JP. Thickness and continuity of the adherent colonic mucus barrier in active and quiescent ulcerative colitis and Crohn's disease. Int J Clin Pract 2008:62:762-9.

36 Fischer $\mathrm{H}$, Stenling R, Rubio $\mathrm{C}$, et al. Differential expression of aquaporin 8 in human colonic epithelial cells and colorectal tumors. BMC Physiol 2001;1:1.

37 Min M, Peng LH, Sun $G$, et al. Aquaporin 8 expression is reduced and regulated by microRNAs in patients with ulcerative colitis. Chin Med J (Engl) 2013:126:1532-7.

38 Yamamoto-Furusho JK, Ascaño-Gutiérrez I, Furuzawa-Carballeda J, et al. Differentia Expression of MUC12, MUC16, and MUC20 in Patients with Active and Remission Ulcerative Colitis. Mediators Inflamm 2015;2015:659018.

39 Wang W, Li Q, Yang T, et al. Expression of AQP5 and AQP8 in human colorectal carcinoma and their clinical significance. World J Surg Oncol 2012;10:242.

40 Matsuyama T, Ishikawa T, Mogushi K, et al. MUC12 mRNA expression is an independent marker of prognosis in stage II and stage III colorectal cancer. Int J Cancer 2010;127:2292-9.

41 Nevalainen TJ, Grönroos JM, Kallajoki M. Expression of group II phospholipase A2 in the human gastrointestinal tract. Lab Invest 1995;72:201-8.

42 Buhmeida A, Bendardaf $R$, Hilska $M$, et al. PLA2 (group IIA phospholipase A2) as a prognostic determinant in stage II colorectal carcinoma. Ann Oncol 2009;20:1230-5.

43 Haapamäki MM, Grönroos JM, Nurmi H, et al. Gene expression of group II phospholipase A2 in intestine in ulcerative colitis. Gut 1997;40:95-101.

44 Lilja I, Dimberg J, Sjödahl R, et al. Effects of endotoxin and dexamethasone on group I and II phospholipase A2 in rat ileum and stomach. Gut 1994;35:40-5.

45 Cooke J, Zhang H, Greger L, et al. Mucosal genome-wide methylation changes in inflammatory bowel disease. Inflamm Bowel Dis 2012;18:2128-37.

46 Lin Z, Hegarty JP, Cappel JA, et al. Identification of disease-associated DNA methylation in intestinal tissues from patients with inflammatory bowel disease. Clin Genet 2011;80:59-67.

47 Kim SW, Kim ES, Moon CM, et al. Abnormal genetic and epigenetic changes in signal transducer and activator of transcription 4 in the pathogenesis of inflammatory bowel diseases. Dig Dis Sci 2012;57:2600-7. 